DOI: https://doi.org/10.46296/ig.v5i9edespfeb.0043

\title{
ESTUDIO DE LA HABITABILIDAD EN LA VIVIENDA DE INTERÉS SOCIAL EN LA PROVINCIA DE MANABÍ.
}

\section{STUDY OF HABITABILITY IN LOW-INCOME HOUSING IN THE PROVINCE OF MANABÍ.}

\author{
Mendoza-Vélez Erika Elizabeth ${ }^{1}$; Ortega-Bravo Bolívar Humberto ${ }^{2}$ \\ ${ }^{1}$ Ingeniera Civil, Universidad Técnica de Manabí, Maestrante del Instituto de Posgrado de la \\ Universidad Técnica de Manabí, UTM. Portoviejo, Ecuador. Correo: \\ emendoza9065@utm.edu.ec. ORCID ID: https://orcid.org/0000-0001-6428-6560 \\ ${ }^{2}$ Máster en Arquitectura del Paisaje, Profesor titular auxiliar de la Universidad Técnica de \\ Manabí, Facultad de Ciencias Matemáticas, Físicas y Químicas. Portoviejo, Ecuador. Correo: \\ bolivar.ortega@utm.edu.ec. ORCID ID: https://orcid.org/0000-0002-1805-8732.
}

\begin{abstract}
Resumen
En el artículo se explora un tema de mucha importancia en lo que respecta al sector de la construcción y a garantizar el acceso a una vivienda de interés social dotada con optimas características de habitabilidad. Poseer una vivienda es una de las necesidades elementales del ser humano; por ello es fundamental el poder conocer si la vivienda de interés social en Manabí cuenta con la referida habitabilidad. Para lo cual la metodología empleada fue mixta, considerando información tanto de campo como bibliográfica; pudiendo así evaluar mediante indicadores de habitabilidad la vivienda de interés social en Manabí. Obteniendo como resultado del estudio que estas casas solo alcanzan una habitabilidad muy básica y totalmente mínima al llegar al 39\% de habitabilidad, lo que demanda que se tomen acciones que mejoren estas características de la vivienda reconsiderando su concepción no solo como un medio para superar el déficit habitacional sino como lo que de ella se requiere para que se constituya en una edificación capaz de satisfacer adecuadamente la demanda de habitabilidad de una familia promedio brindando confort y seguridad.
\end{abstract}

Palabras clave: Vivienda de interés social, habitabilidad, confort, seguridad, déficit habitacional.

\begin{abstract}
The article explores an issue of great importance in regards to the construction sector and to guarantee access to affordable housing with optimal habitability characteristics. Owning a home is one of the basic needs of the human being; For this reason it is essential to be able to know if the social housing in Manabí has the referred habitability. For which the methodology used was mixed, considering both field and bibliographic information; Thus, it is possible to evaluate lowincome housing in Manabí through habitability indicators. Obtaining as a result of the study that these houses only achieve a very basic and totally minimal habitability when reaching $39 \%$ of habitability, which demands that actions be taken to improve these characteristics of the house, reconsidering its conception not only as a means to overcome the housing deficit but as what is required of it to become a building capable of adequately meeting the demand for habitability of an average family providing comfort and security.
\end{abstract}

Keywords: Low-income housing, habitability, comfort, security, housing deficit.

Información del manuscrito:

Fecha de recepción: 21 de diciembre de 2021.

Fecha de aceptación: 01 de febrero de 2022.

Fecha de publicación: 07 de febrero de 2022. 


\section{Introducción}

Una de las grandes deudas políticas y sociales desde siempre ha sido brindar una respuesta al creciente déficit habitacional el mismo que se incrementa cada año y que hoy en día según las cifras oficiales se encuentra alrededor de 1,7 millones de unidades habitaciones, es decir, 1,7 millones de familias que no poseen vivienda propia y que viven en condiciones deplorables como el hacinamiento que es muy frecuente cuando una misma vivienda es ocupada por tres generaciones (los abuelos, los padres, hijos y nietos) es así como en viviendas de menos de 50 metros cuadrados llegan a ser habitadas por más de 10 personas en sitios que no gozan de las prestaciones de seguridad $y$ servicios básicos necesarios.

Esta situación ha incidido en que los gobiernos a través del MIDUVI planten soluciones habitaciones las cuales presentan una disposición demasiado básica e inclusive precaria lo que genera la necesidad de estudiar profundamente la habitabilidad de estas unidades; ante lo cual se realizó una búsqueda exhaustiva de estudios relacionados obteniendo los datos siguientes:
En un estudio realizado en México en relación a la medición de la habitabilidad de la vivienda de interés social. Caso de estudio: Municipio de Ecatepec y Tecámac; con una metodología de índole histórica contextual se planteó la conclusión siguiente: "la vivienda construida en 1989 logra alcanzar 67 puntos, mientras que las viviendas edificadas entre una década y dos después, comparten un estándar más bajo en cuanto a la habitabilidad: las construidas en el año 2000 alcanzan una calificación de 53 puntos; y las viviendas del 2012 es de 55 puntos" (Rendón, 2019).

También se colige el estudio denominado: ¿Cómo es la habitabilidad en viviendas de interés social? Caso de estudio: fraccionamientos Lomas Del Bosque y Privadas la Torre En Saltillo, Coahuila; en el cual se indica como conclusión que: "lo más importante en la vivienda es resolver las circulaciones, número de espacios y m2 construidos. Además, se debe preocupar en obtener satisfacción, privacidad y funcionalidad, las dos primeras relacionadas con el diseño 
de espacios y conexiones" (Molar \& Aguirre, 2013)

Asimismo, este tipo de estudio también se ha en el contexto nacional con tema: La habitabilidad en la vivienda social en Ecuador a partir de la visión de la complejidad: elaboración de un sistema de análisis; en este estudio de carácter bibliográfico, se llegó a concluir que: "habrá una directa influencia de la vivienda en el ser humano, sin embargo, habrá que considerar también cómo el ser humano transforma y se adapta a la vivienda. Por otro lado, hemos explicado que en una visión más amplia la vivienda agrupa cualidades físicas y sociales que serán las que le confieren las condiciones óptimas de habitabilidad" (Rodas, 2018).

También el trabajo realizado en la ciudad de Ambato de tema "funcionalidad de los espacios interiores de la vivienda social en Ecuador" siendo un estudio tanto bibliográfico como de campo en el mismo que se establece la siguiente conclusión: "Se enfatizó sobre los espacios con los que podría contar la vivienda social desde la participación comunitaria en base a los requerimientos y necesidades de sus habitantes con el plus de expandirse y formar nuevos núcleos sociales" (Naula, 2018)

Como vemos cada uno de los estudios en mención se constituye un antecedente de gran importancia a la presente investigación en base a las necesidades identificadas en la habitabilidad de la vivienda de interés social.

El estudio de la habitabilidad de la vivienda de interés social es un tema de suma importancia ya que a la hora de atender este tipo de necesidad se traduce en identificar cuantas viviendas faltan y tratar de brindar una solución en términos numéricos; pero lo que se evidencia es que cada vez la vivienda de interés social va disminuyendo su calidad y su espacio y aun así los gobiernos son incapaces de cubrir el déficit existente.

Por lo tanto en términos de eficiencia al no estar en condiciones de solventar todo el número de unidades habitaciones necesarias, sería más conveniente el dotar de viviendas con un alto índice de habitabilidad que cubra mejor las necesidades de sus ocupantes 
dotadas de un espacio adecuado de buena iluminación, instalaciones debidas, un buen nivel de salubridad, dotadas de elementos de fácil aseo, materiales en los cuales no proliferen fácilmente bacterias microrganismos.

Por lo tanto, los beneficios de la presente investigación están direccionados a visibilizar las condiciones de habitabilidad de las viviendas de interés social, así como este aspecto incide en el bienestar de sus ocupantes; con la finalidad de que se puedan tomar acciones para mejorar las condiciones de habitabilidad.

Las condiciones de habitabilidad de la vivienda de interés social deben ser adecuadas para albergar apropiadamente a una familia promedio que puede ir de 4 a 8 ocupantes brindándoles espacio suficiente para el desarrollo de sus actividades cotidianas, debemos destacar que las familias a las que van direccionadas este tipo hogar son familias en estado de pobreza y vulnerabilidad económica e incluso en ocasiones sus integrantes pueden presentar alguna discapacidad, por lo cual no es adecuado brindar una vivienda impropia esperando que el grupo familiar mejores por su cuenta las condiciones de habitabilidad de la vivienda, es algo que no va a suceder a corto plazo, por ello se requiere de este estudio a fin de profundizar en las condiciones de habitabilidad deseadas para una vivienda de interés social.

La vivienda de interés social, durante los años ha sido una necesidad básica que debe satisfacerse en cualquier parte del mundo (Zimbrón \& Toledo, 2011). En el Ecuador el Ministerio de Desarrollo Urbano y Vivienda (MIDUVI), con la finalidad de llegar a cumplir lo estipulado en el artículo 30 de la Constitución y los distintos acuerdos ministeriales, en donde se ha llegado a determinar que los programas de vivienda de interés social están orientados a "facilitar el acceso a una vivienda y que cumpla con condiciones de habitabilidad y servicios básicos indispensables" (Báez Gordillo, 2017).

El incremento poblacional y déficit habitacional en el Ecuador ha generado la dotación de vivienda de insuficiente habitabilidad para la población, es entonces donde cobra gran importancia el concepto de una 
vivienda digna para brindar un estado de dignidad a los integrantes de la familia con los espacios adecuados para su desarrollo.

Conociendo el déficit de vivienda a nivel nacional y como deber primordial de erradicar la pobreza y promover el progreso económico, social y cultural del país, diferentes organismos ya sean públicos 0 privados han iniciado un proceso de crecimiento del área inmobiliaria orientado a atender el segmento de "Vivienda Social". De conformidad con las políticas del Gobierno Nacional y para servir a la población más vulnerable, pobre y desposeída del país, fue necesario introducir nuevos parámetros normativos a la reglamentación existente del Sistema de Incentivos para la Vivienda urbana, rural y urbano marginal, que respondan a la real situación económica de los habitantes.

Por tal razón para asegurar que la vivienda social cumpla con los objetivos constitucionales de ser digna para la población, se considera prioritario la habitabilidad. Lo que permitirá contar con un instrumento que facilite el realizar este análisis.
En la provincia de Manabí el sector más pobre y desposeído de la población a menudo se encuentra en los barrios periféricos urbanos marginales un pequeño porcentaje de ellos han accedido al beneficio de una vivienda de interés social, construida a través del MIDUVI muchas de las cuales tienen un área de construcción muy reducida que no supera los $45 \mathrm{~m} 2$ cuentan con un solo baño, las instalaciones de la cocina son muy reducidas sin espacios para guardar los alimentos, las paredes así como el techo a su vez tampoco presentan aislamiento térmico, entre otras deficiencias que afectan el grado de habitabilidad de la vivienda de interés social; lo que nos lleva al siguiente cuestionamiento ¿Cuál es la habitabilidad de la vivienda de interés social en la provincia de Manabí?

Por ello se establece como objetivo: Analizar el nivel habitabilidad de la vivienda de interés social, en la provincia de Manabí.

De la misma manera se trazaron los siguientes objetivos: 
- Caracterizar la habitabilidad básica requerida para la vivienda de interés social.

- Evaluar el nivel de habitabilidad de la vivienda de interés social en la provincia de Manabí.

- Determinar conclusiones fundamentales en relación a la evaluación realizada al nivel de habitabilidad de la vivienda de interés social en la provincia de Manabí.

Asimismo, la hipótesis es: El nivel de habitabilidad de la vivienda de interés social en la provincia de Manabí no es adecuado a las necesidades de sus ocupantes.

\section{DESARROLLO.}

\section{Habitabilidad.}

"La habitabilidad corresponde a las características y cualidades del espacio, entorno social y medio ambiente, de una edificación en este caso de la vivienda de interés social que contribuyen singularmente a brindar a sus ocupantes una sensación de bienestar y seguridad" (ONU, 2020). Por lo tanto, la vivienda de interés social debe contar una habitabilidad adecuada la misma que ofrezca condiciones idóneas a las familias que las ocupan y esto actualmente se ha venido a constituir en una necesidad del desarrollo urbano a nivel mundial dado el incremento de los asentamientos urbanos en la mayoría de las ciudades a nivel mundial.

Características de la habitabilidad.

(Mercado, 2015) sostiene deben existir tres características mínimas para la habitabilidad de una edificación o vivienda:

$>$ Subjetividad y percepción de los sujetos.

$>$ Factores externos provenientes de la sociedad y las condiciones ambientales.

Adaptabilidad de los diseños a estos puntos.

La habitabilidad se confiere a la relación de los habitantes con la vivienda, como un escenario de interacción humana más antiguo e importante lugar en el que se desarrolla el núcleo de la sociedad que es la familia, por ello su trascendental importancia a nivel social $y$ en lo referente a los estándares de la creación de la obra civil (Mercado, 2015). 
Importancia de la habitabilidad.

La vivienda de interés social tiene gran importancia debido a que es la vía más adecuada para otorgar una solución habitacional a las familias de más bajas condiciones económicas, las cuales no pueden acceder a una vivienda que les garantice seguridad y bienestar, la vivienda de interés social es un medio para fortalecer el desarrollo urbano planificado mediante hogares provistos de seguridad, salubridad, cercanos a centros de recreación, así como a centros de salud, entre otros servicios necesarios para el bienestar familiar y comunitario.

\section{Cualidades de la habitabilidad.}

La habitabilidad concurre con las condiciones que se establecen como ideales o mínimamente deseables para el desarrollo de la familia y sus actividades cotidianas, así como de interacción social, (Bernal, 2015) señala "la habitabilidad es una condición de tangible, como cualitativa, que se relaciona con el ser del hombre, son cualidades que deben facilitar al ser humano para el correcto desarrollo social y económico de la familia".
De acuerdo con esto, la existencia de una familia tiene un sistema de relaciones con el entorno construido que necesariamente obedece al desarrollo urbano sostenible en un marco de coherencia y de bienestar para ello las edificaciones deben proveer la habitabilidad necesaria a sus ocupantes.

Otra cualidad fundamental supone que la habitabilidad es una acción cuantitativa relacionada directamente con la calidad de vida siendo así es posible cuantificar mediante estándares numéricos y porcentuales las condiciones deseadas de habitabilidad para el correcto crecimiento de las comunidades así como de las familias que las integran estos estándares son incorporados a la legislación correspondiente en los diferentes cuerpos legales y normativos, así como a las entidades que deben resguardar y garantizar su cumplimiento las que en nuestra realidad son las municipalidades mediante los departamentos designados para esta finalidad.

Factores de habitabilidad.

La habitabilidad es inherente a las cualidades de construcción, así 
como de diseño de las edificaciones y viviendas por lo cual (Mercado, 2015) establece los siguientes factores:

Dimensiones físicas de la vivienda. El tamaño de la vivienda debe estar pensado para que el espacio sea suficiente para garantizar el adecuado desarrollo de una familia tipo de mínimo cinco miembros.

Conectividad. Relación que hay entre los espacios, facilidad de acceso, para que los ocupantes se puedan desplazar de manera adecuada.

Circulación. Tanto en el sentido vertical como horizontal la circulación es fundamental aún más si existen personas con capacidades especiales en las familias.

Seguridad: se relaciona directamente con los materiales de construcción de la vivienda el cumplimiento de normativas antisísmicas y de prevención de siniestros; así como también que este dotada de puertas, ventanas y cerramientos que impidan en lo posible que se de algún ataque o robo a la vivienda.

> Operatividad. Grado de satisfacción que tiene un espacio para realizar las actividades a la que está destinada como que los sanitarios cuenten con un espacio adecuado para realizar las necesidades fisiológicas, que la cocina cuente con las dimensiones favorables para los enceres, alacenas, fregaderos, estufa, nevera y espacio suficiente para cocinar, las habitaciones deben estar provistas de área suficiente para el descanso y provista de guardarropas y mesa de noche. Confortabilidad. Que todos los miembros de la familia se sientan cómodos con el espacio suficiente para llevar a cabo sus actividades cotidianas sintiéndose en un ambiente de bienestar.

Privacidad. En una vivienda se realizan actividades que requieren de intimidad, pensando en ello la vivienda debe proveer de privacidad a sus ocupantes que en ocasiones pueden estar sin 
vestimenta al cambiarse de ropa o de asearse.

La vivienda de interés social

La vivienda social requiera de calidad, debe ser una solución eficiente, una alternativa de producción en base a una tecnología constructiva que considere las necesidades de sus ocupantes; que, propiciando la incorporación de sus habitantes en el desarrollo, selección, e implementación de las viviendas siendo así una solución tanto de habitabilidad como en la creación de empleos en los sectores más desposeídos (Fermin, 2015).

No debe ser una solución provisoria para la familia, la vivienda de interés social debe tener características de durabilidad y eficiencia en su diseño y construcción debe estar dotada de todos los servicios para $y$ comodidades básicas para sus habitantes; debe ser una vivienda digna, sana, con agua, alcantarillado y luz (Ortiz, 2017).

Por otra parte, la vivienda de interés social debe disponer de una arquitectura capaz de adaptarse a las necesidades culturales $y$ económicas de sus habitantes teniendo establecido que es una familia con requerimientos $y$ necesidades (Fermin, 2015).

Características de la vivienda de interés social.

La mayoría de los programas desarrollados por MIDUVI han tomado como base dos tipos de proyectos que se repiten, fundamentalmente en áreas rurales y áreas urbanas periféricas. El primer prototipo consiste en una vivienda de $42 \mathrm{~m} 2$, de planta cuadrada en dos crujías, con dos habitaciones, un baño y un espacio integrado de sala y comedor, cocina, con cubierta ligera de planchas acanaladas galvanizadas, 3 o 4 ventanas cuadradas de vidrio de corredera, ubicadas solo en las paredes de frente $y$ fondo. Las condiciones térmicas interiores se agravan por la ausencia de aleros 0 portales.

Los proyectos de vivienda deben contar con procesos de densificación planificados, adecuadas condiciones de calidad, de áreas comunes, ser accesibles a la población de bajos recursos.

Las iniciativas desarrolladas por el MUDUVI desde el año 1961 guarda características de una vivienda en 
hilera de una sola planta en dos crujías, paredes de bloque enlucidas en cemento caída a dos aguas con patio frontal y posterior.

Ilustración 1. Vivienda de interés social promovida por el MIDUVI.
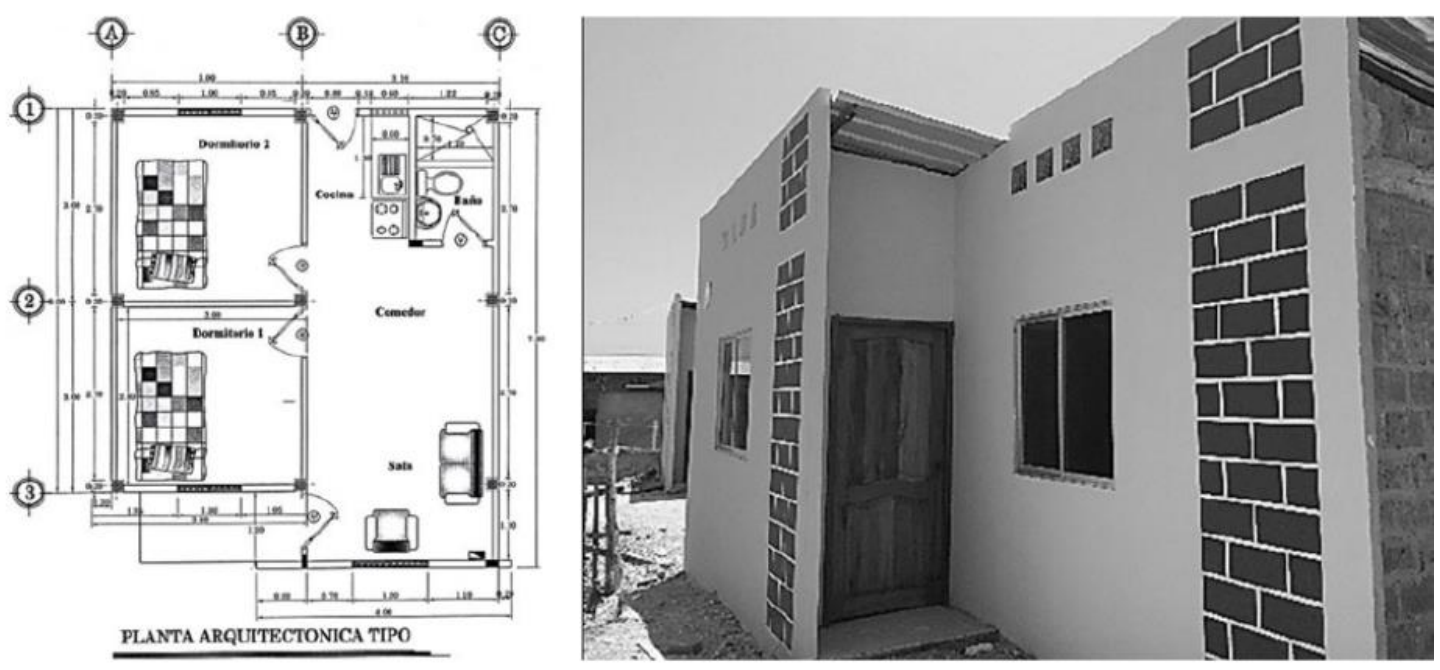

Fuente: (Véliz, 2019)

Importancia de la vivienda de interés social.

Un derecho a un lugar que satisface insuficiencias básicas es lo contempla una vivienda, necesidad que esta tutelada en la Constitución Política del Ecuador en su Art. 30. Pero la vivienda es más que esto, se trata de un resguardo donde la familia encuentra seguridad y confort para llevar una vida decorosa y digna, sin padecer de las inclemencias ambientales, o los riesgos ocasionados por el hombre como la delincuencia.

La vivienda de interés social nace de la iniciativa gubernamental de dotar de una casa a las familias de escasos recursos económicos, una edificación realizada en hormigón dotada de áreas suficientes para brindar abrigo y seguridad a una familia compuesta en promedio por cinco personas. La importancia de la vivienda de interés social y el poder acceder a ella se encuentra reconocida como un derecho humano fundamental.

Es un espacio en el cual se encuentra el descanso, donde almacenar y prepara los alimentos satisfacer las necesidades básicas inherentes al ser humano, un lugar en el cual conformar una familia de forma digna. 
Demanda de la vivienda de interés social.

Según indica el BI (2019) "sostiene que más de dos millones de familias en Ecuador se encuentran en déficit de vivienda y más de un millón de estas familias se encuentran en las zonas urbanas" causando que estas familias se encuentren en estado de vulnerabilidad debido a sus características socioeconómicas las cuales en muchas ocasiones les impide acceder al pago de rubro de alquileres de inmuebles adecuados con características de habitabilidad que ofrezcan un sano desarrollo.

Por su parte el INEC (2020) es más conservador en sus cifras "por lo que establece que el déficit de unidades habitaciones se encuentran en 1,7 millones de unidades". Sin embargo, se observa que el déficit habitacional de ecuatorianos que no pueden acceder a una vivienda propia no es el único problema relacionado a las necesidades de la población respecto de su hogar, ya que el problema cualitativo es más profundo puesto que millones de familias a pesar de tener una vivienda esta no cuenta con las características de una vivienda digna, en muchos caso son edificaciones que carecen de un diseño estructural que brinde garantías, los materiales suelen ser mixtos y sensibles a incendios $y$ siniestros al mismo tiempo carecen de instalaciones de alcantarillado, agua potable, alumbrado público incluso hasta de electricidad en muchos casos, es preciso que estos casos puedan ser cuantificados para conocer de forma real las condiciones de los hogares de los ecuatorianos y poder establecer medidas que permitan aplacar esta realidad que es padecida por un sinnúmero de familias.

\section{Materiales y métodos}

La metodología empleada en el presente estudio para la medición de la habitabilidad de la vivienda de interés social en Manabí se empleó el método propuesto por Abadi y Martín (Abadi Abbo \& Martín Domínguez, 2009). Con el cual realizaron la evaluación a viviendas de interés social en Caracas, Venezuela, analizando el estado y estructura física de las viviendas y las actividades que se realizan en ellas, de la misma manera se incluyen factores o indicadores que 
son exógenos pero que sin duda inciden considerablemente en las condiciones de habitabilidad de la vivienda ya que atañen al estado de bienestar de los ocupantes de la vivienda de interés social.

Siendo así que en el método se considera la medición de 40 variables, sin embargo, para el presente estudio se priorizaron 38 los cuales se las considero como las variables de más incidencia en la habitabilidad de la vivienda de interés social para la provincia de Manabí.

En esta metodología existen tres categorías de puntaje con la cual se pondera:

Tabla 1. Criterio de ponderación.

\begin{tabular}{|l|l|}
\hline 0 & No cumplen con las condiciones mínimas \\
\hline 1 & Cumple con lo mínimo \\
\hline 2 & $\begin{array}{l}\text { Cumple con lo mínimo que marca la norma, además de proponer alguna } \\
\text { solución extra a las necesidades }\end{array}$ \\
\hline 3 & $\begin{array}{l}\text { Cumple con todos los parámetros, tanto de las normas consideradas, como de } \\
\text { manuales de diseño arquitectónico general e incluso es superado }\end{array}$ \\
\hline
\end{tabular}

Fuente: Abadi Abbo \& Martín Domínguez, 2009.

Al finalizar la valoración se procede

a realizar una sumatoria la misma que goza de un porcentaje en relación al puntaje máximo que es
121, por tratarse de 38 variables o aspectos.

Indicadores:

Tabla 2. Indicadores considerados para la evaluación de la VIS.

\begin{tabular}{|l|l|l|l|}
\hline 1 & $\begin{array}{l}\text { Tipología de la vivienda (aislada, } \\
\text { pareadas o continuas) }\end{array}$ & 2 & Superficie del lote \\
\hline 3 & Ancho de lote & 4 & Área de construcción \\
\hline 5 & Acceso peatonal a la vivienda & 6 & Estacionamiento \\
\hline 7 & $\begin{array}{l}\text { Posibilidad y previsión de } \\
\text { crecimiento de la vivienda. }\end{array}$ & 8 & Componentes espaciales de la vivienda \\
\hline 9 & Estares separados & 10 & Comedores separados \\
\hline 11 & $\begin{array}{l}\text { Estares y comedores integrados o } \\
\text { separados }\end{array}$ & 12 & Cocina \\
\hline 13 & Área de servicio-lavado y secado & 14 & Dormitorios / Dormitorios dobles 1 o 2 \\
\hline 15 & Sanitario & 16 & Área de almacenaje \\
\hline 17 & Circulaciones internas & 18 & Depósito de basura \\
\hline 19 & Altura mínima en los espacios & 20 & $\begin{array}{l}\text { lluminación natural de los espacios de la } \\
\text { vivienda }\end{array}$ \\
\hline 21 & $\begin{array}{l}\text { Asoleamientos de los espacios de } \\
\text { la vivienda }\end{array}$ & 22 & $\begin{array}{l}\text { Ventilación natural de los espacios de la } \\
\text { vivienda }\end{array}$ \\
\hline 23 & $\begin{array}{l}\text { Protecciones ambientales, aleros, } \\
\text { aceras }\end{array}$ & 24 & lluminación artificial y toma corrientes X \\
\hline
\end{tabular}




\begin{tabular}{|l|l|l|l|}
\hline 25 & Instalaciones sanitarias & 26 & $\begin{array}{l}\text { Drenaje de aguas de lluvia: techos, } \\
\text { patios, retiros }\end{array}$ \\
\hline 27 & $\begin{array}{l}\text { Otros servicios: teléfono, } \\
\text { televisión, recolección de basura }\end{array}$ & 28 & $\begin{array}{l}\text { Sistema constructivo: eficiencia y } \\
\text { durabilidad de los materiales y los } \\
\text { acabados }\end{array}$ \\
\hline 29 & Privacidad interna y externa & 30 & Apariencia externa de la vivienda \\
\hline 31 & Tratamiento de áreas exteriores & 32 & Seguridad y Riesgo en la construcción \\
\hline 33 & $\begin{array}{l}\text { Seguridad contra robo, vandalismo, } \\
\text { acceso de animales indeseables }\end{array}$ & 34 & $\begin{array}{l}\text { Distancia a servicios escolares: } \\
\text { maternales, preescolar y escuelas }\end{array}$ \\
\hline 35 & $\begin{array}{l}\text { Distancia a parques: infantiles, } \\
\text { áreas recreacionales, canchas } \\
\text { deportivas }\end{array}$ & 36 & $\begin{array}{l}\text { Distancia a comercios locales: abastos, } \\
\text { farmacias, panaderías }\end{array}$ \\
\hline 37 & $\begin{array}{l}\text { Distancia a ambulatorios o centros } \\
\text { de salud }\end{array}$ & 38 & Distancia a paradas de transporte \\
\hline
\end{tabular}

Fuente: Abadi Abbo \& Martín Domínguez, 2009.

La población y muestra de la presente investigación consistió en la aplicación del instrumento de evaluación de la habitabilidad a 100 viviendas de interés familiar consideradas de manera aleatoria entre los cantones Jaramijó, Portoviejo, Rocafuerte y Chone, en la iniciativas construidas por el MIDUVI, "casa para todos" así como también las que han sido realizadas en terrenos particulares con financiación del MIDUVI, por facilidad logística las viviendas consideras se mantienen dentro del perímetro urbano de cantón a fin de que esto sea establecido como un criterio constante del presente estudio. 


\section{Resultados y discusión}

Tabla 3. Resultados de la evaluación de habitabilidad de la VIS en Manabí.

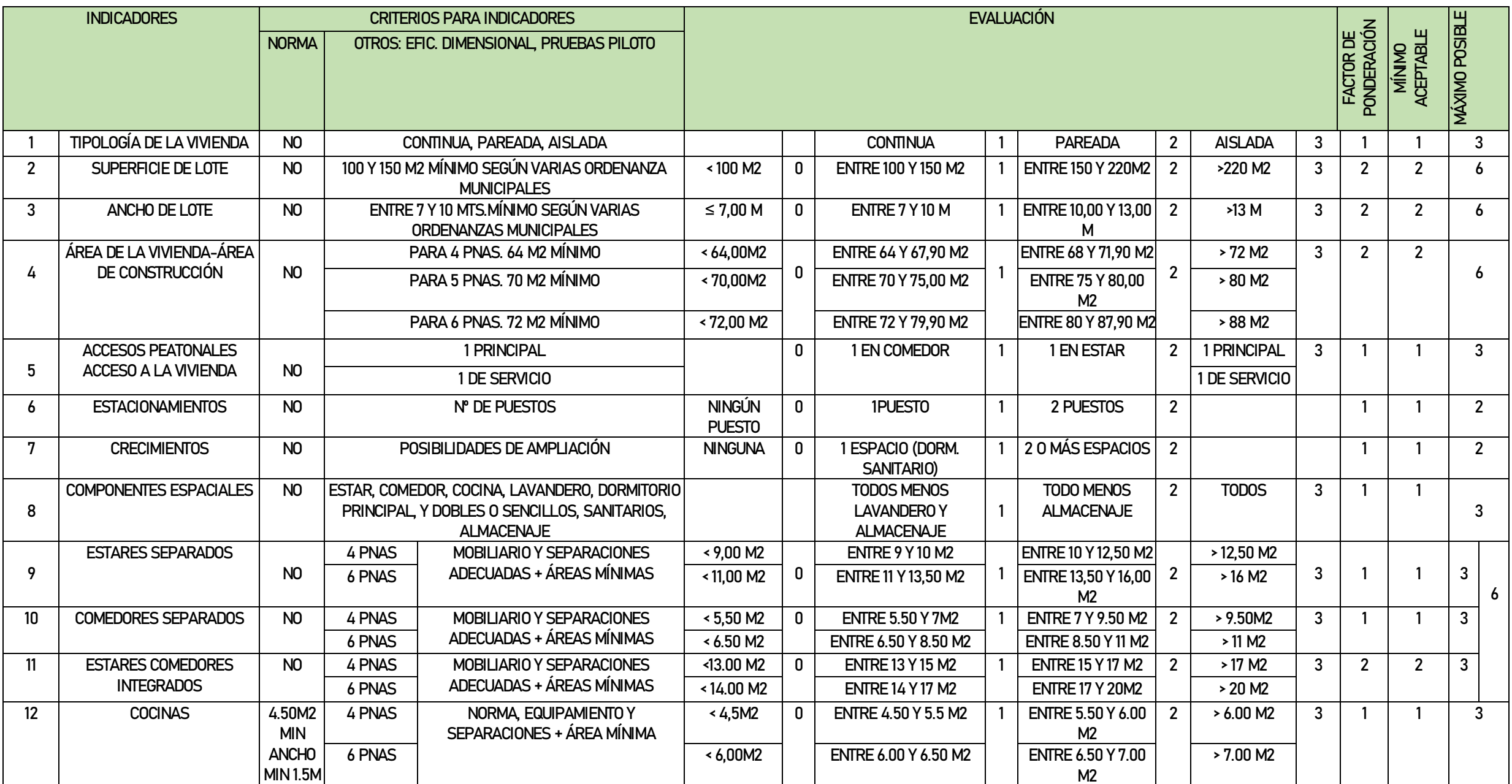




\begin{tabular}{|c|c|c|c|c|c|c|c|c|c|c|c|c|c|c|c|}
\hline 13 & ÁREA DE SERVICIO LAVADERC & \begin{tabular}{|l|}
$3.00 \mathrm{M} 2$ \\
MÍNIMO \\
\end{tabular} & $\begin{array}{l}\text { NORMAY EQUIPAMIENTO, SEPARACIONES } \\
\text { ADECUADAS + ÁREA MÍNIMA. }\end{array}$ & \begin{tabular}{|c|}
$<3.00 M 20$ \\
INEXISTENTE
\end{tabular} & 0 & ENTRE 3.00 Y 4.5 M2 & 1 & ENTRE 4.5 Y $5.50 \mathrm{M} 2$ & 2 & $>5.50 \mathrm{M} 2$ & 3 & 1 & 1 & 3 & 3 \\
\hline 14 & DORMITORIO PRINCIPAL & $\mid \begin{array}{c}9.00 \mathrm{M} 2+ \\
\text { CLOSET }\end{array}$ & $\begin{array}{l}\text { NORMA Y EQUIPAMIENTO, SEPARACIONES } \\
\text { ADECUADAS + ÁREA MÍNIMA Y ANCHO MÍNIMO. }\end{array}$ & $\begin{array}{l}<9.00 \mathrm{M} 2+ \\
\text { CLOSET, } \\
2.40 \mathrm{M} \mathrm{DE} \\
\text { ANCHO }\end{array}$ & 0 & $\begin{array}{c}9.00 \mathrm{M} 2+\text { + CLOSET, } \\
\text { ANCHO MÍNIMO } 2.40 \mathrm{M}\end{array}$ & 1 & $\begin{array}{c}\text { ENTRE } 9.00 \text { Y } 9.5 \mathrm{M} 2 \\
+ \text { CLOSET, ANCHO } \\
\text { MÍNIMO } 2.90 \mathrm{M}\end{array}$ & 2 & \begin{tabular}{|c}
$>9.5 \mathrm{M} 2+$ \\
CLOSETY \\
ANCHO MINIMO \\
$3.00 \mathrm{M}$
\end{tabular} & 3 & 1 & 1 & 3 & 9 \\
\hline 14 & DORMITORIOS DOBLES 102 & $\left|\begin{array}{c}8.00 \mathrm{M} 2+ \\
\text { CLOSET, }\end{array}\right|$ & $\begin{array}{l}\text { NORMA Y EQUIPAMIENTO, SEPARACIONES } \\
\text { ADECUADAS + ÁREA MÍNIMA Y ANCHO MÍNIMO. }\end{array}$ & $\begin{array}{l}<8.00 \mathrm{M} 2+ \\
\text { CLOSET, } \\
2.40 \mathrm{M} \mathrm{DE} \\
\text { ANCHO }\end{array}$ & 0 & $\begin{array}{c}8.00 \mathrm{M} 2+\text { CLOSET, } \\
\text { ANCHO MINIMO } 2.40 \mathrm{M}\end{array}$ & 1 & $\begin{array}{c}\text { ENTRE } 8.00 \text { Y } 8,5 \\
\text { M2,+ CLOSET, } \\
\text { ANCHO MÍNIMO } 2.80 \\
\text { M }\end{array}$ & 2 & \begin{tabular}{|c}
$>8,5 \mathrm{M} 2+$ \\
CLOSET Y \\
ANCHO MINIMO \\
$3.00 \mathrm{M}$
\end{tabular} & 3 & 1. & 1 & $\begin{array}{c}30 \\
6\end{array}$ & \\
\hline 14 & DORMITORIOS INDIVIDUALES & $\begin{array}{c}6 \mathrm{M} 2+ \\
\text { CLOSET }\end{array}$ & $\begin{array}{c}\text { NORMA Y EQUIPAMIENTO, SEPARACIONES } \\
\text { ADECUADAS + ÁREA MÍNIMA Y ANCHO MÍNIMO. }\end{array}$ & $\begin{array}{c}<6.0 \mathrm{M} 2+ \\
\text { CLOSET, } \\
1.50 \mathrm{M} \text { DE } \\
\text { ANCHO }\end{array}$ & 0 & $\begin{array}{c}\text { 6.0 M2 + CLOSET, ANCHO } \\
\text { MÍNIMO } 2.00 \mathrm{M}\end{array}$ & 1 & $\begin{array}{c}\text { ENTRE 6.0 Y 8.00 } \\
\text { M2,+ CLOSET, } \\
\text { ANCHO MÍNIMO 2.30 } \\
\text { M }\end{array}$ & 2 & \begin{tabular}{|c|}
$>8,00 M 2+$ \\
CLOSETY \\
ANCHO MINIMO \\
$2.30 \mathrm{M}$
\end{tabular} & 3 & 1 & 1 & $\begin{array}{c}00 \\
3\end{array}$ & \\
\hline 15 & SANITARIOS & \begin{tabular}{|c|}
$2.88 \mathrm{M} 2$ \\
ANCHO \\
MíNIMO \\
1.20 \\
\end{tabular} & $\begin{array}{c}\text { NORMA, EQUIPAMIENTO, SEPARACIONES ADECUADAS } \\
\text { MÁS ÁREA Y ANCHO MÍNIMO }\end{array}$ & $\begin{array}{l}<2,88 \mathrm{M} 2 \mathrm{Y} \\
1,20 \mathrm{M} \mathrm{DE} \\
\mathrm{ANCHO}\end{array}$ & 0 & $\begin{array}{c}\text { ENTRE 2,88Y 3,20 M2Y } \\
\text { 1,20 M DE ANCHO }\end{array}$ & 1 & \begin{tabular}{|c|} 
ENTRE 3,20 Y 3,80 \\
M2 Y ANCHO > 1,20 M
\end{tabular} & 2 & $\begin{array}{c}>3,80 \mathrm{M} 2 \mathrm{Y} \\
\mathrm{ANCHO} \geq 1,40 \\
\mathrm{M}\end{array}$ & 3 & 1 & 1 & 3 & 3 \\
\hline 16 & ÁREAS DE ALMACENAJE & NO & $\begin{array}{l}\text { 0,5 ML/PNA; DE LOS CLOSETS DE DORMITORIOS, SI } \\
\text { SOBRAN DE 0.80 ML/PNA SE CONSIDERA ÁREA DE } \\
\text { ALMACENAJE }\end{array}$ & NO TIENE & 0 & $<1 \mathrm{ML}$ & 1 & ENTRE 1Y1,90 ML & 2 & $\geq 2 \mathrm{ML}$ & 3 & 1 & 1 & 3 & 3 \\
\hline 17 & CIRCULACIONES INIERNAS & $\begin{array}{c}\text { 900MM } \\
\text { ACCESIBI } \\
\text { LIDAD }\end{array}$ & CONFINADAS ANCHO MINIMO 0,90 (ENIRE PAREDES) & $<0,90$ & 0 & ENTRE 0,90 Y $1.10 \mathrm{M}$ & 1 & ENTRE 1,10 Y 1,20 M & 2 & $\geq 1,20 \mathrm{M}$ & 3 & 1 & 1 & 3 & 3 \\
\hline 18 & DEPÓSITO DE BASURA & NO & ÁREAS: ANCHOS MÍNIMOS 0,60 M & NO TIENE & 0 & ENTRE 0,60 Y 1,00 M2 & 1 & ENTRE 1Y 1,50 M2 & 2 & $\geq 1,50 \mathrm{M} 2$ & 3 & 1 & 1 & 3 & 3 \\
\hline 19 & ALTURAS INTERIORES & $\mid \begin{array}{c}2,50 M \\
\text { NEC } \\
\text { VIVIENDA } \\
\text { S HASTA } \\
2 \text { PISOS }\end{array}$ & $\begin{array}{l}\text { ALTURAS MÍNIMASY PROMEDIOS ENTECHOS } \\
\text { INCLNADOS 2,5 ESP. DE HAB. 2,40 BAÑOS }\end{array}$ & $\begin{array}{l}<2,50 \mathrm{Y} \\
2,40 \mathrm{BANNOS}\end{array}$ & 0 & $\begin{array}{l}2.50 \text { ESP. HAB. } 02,40 \\
\text { BAÑOS }\end{array}$ & 1 & $>2,50 \mathrm{M}$ & 2 & & & 1 & 1 & 2 & 2 \\
\hline 20 & ILUMINACIÓN NATURAL & $\begin{array}{c}10 \% \\
\text { SUPERF. } \\
\text { DEL } \\
\text { ESPACIO }\end{array}$ & $\begin{array}{l}\text { PORCENTAJE DEL ÁREA DEL ESPACIO; POSICIÓN DE } \\
\text { VENTANA E ILUMINACIÓN BIEN DISTRIBUIDA }\end{array}$ & $<10 \%$ & 0 & $\begin{array}{c}\geq 10 \% \text { ILUMINACIÓN MAL } \\
\text { DISTRIBUIDA }\end{array}$ & 1 & \begin{tabular}{|c|}
$\geq 10 \%$ ILUMINACIÓN \\
BIEN DISTRIBUIDA
\end{tabular} & 2 & & & 1 & 1 & 2 & 2 \\
\hline
\end{tabular}




\begin{tabular}{|c|c|c|c|c|c|c|c|c|c|c|c|c|c|c|}
\hline \multirow{2}{*}{\multicolumn{2}{|c|}{ INDICADORES }} & \multirow{2}{*}{\multicolumn{2}{|c|}{ CRITERIOS PARA INDICADORES }} & \multirow{2}{*}{\multicolumn{8}{|c|}{ EVALUACIÓN }} & \multirow{3}{*}{ 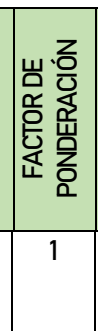 } & \multirow{3}{*}{ 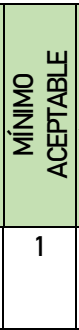 } & \multirow{3}{*}{ 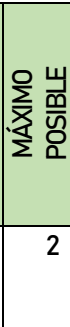 } \\
\hline & & & & & & & & & & & & & & \\
\hline 21 & ASOLAMIENTO DE LOS ESPACIOS & \begin{tabular}{c|} 
NORMA \\
\\
NO
\end{tabular} & \begin{tabular}{|c} 
OTROS: EFC. DIMENSIONAL, PRUEBAS \\
PILOTO \\
PROTEGER DEL OESTEY SUROESTE
\end{tabular} & \begin{tabular}{|c|} 
NINGUNA \\
PROTECCIÓN
\end{tabular} & 0 & \begin{tabular}{|c|} 
ESTAR COMEDOR \\
PROTEGIDOS
\end{tabular} & 1 & $\begin{array}{l}\text { ESTAR, COMEDORY } \\
\text { DORMITORIO PPAL } \\
\text { PROTEGIDOS } \\
\end{array}$ & 2 & & & & & \\
\hline 22 & VENTILACION NATURAL & $\begin{array}{l}\text { 10\% ÁREA DE } \\
\text { ESPACIOS }\end{array}$ & $\begin{array}{l}\text { \% DEL ÁREA DE ESPACIOS. USO DE } \\
\text { LUCERNARIOS PARA VENTILAR }\end{array}$ & $<10 \%$ & 0 & $\begin{array}{l}\geq 10 \% \text { ESPACIO } \\
\text { VENTLADO CON } \\
\text { LUCERNARIOS } \\
\end{array}$ & 1 & \begin{tabular}{c|}
$\geq 10 \%$ SIN \\
LUCERNARIO
\end{tabular} & 2 & & & 1 & 1 & 2 \\
\hline 23 & $\begin{array}{c}\text { PROTECCIONES AMBIENTALES. } \\
\text { ALEROS, ACERAS }\end{array}$ & NO & CONIRA ШUVIA, INUNDACIÓN & NO TIENE & 0 & $\begin{array}{c}\text { ACERAS O ALEROS } \\
\leq 20 \mathrm{CM}\end{array}$ & 1 & $\begin{array}{c}\text { ACERASY ALEROS } \\
>30 \mathrm{CM}\end{array}$ & 2 & & & 1 & 1 & 2 \\
\hline 24 & ILUMINACIÓNYTOMAS & $\begin{array}{c}\text { No DE LUX POR } \\
\text { AMBIEN }\end{array}$ & $\begin{array}{c}100 \text { LUX: ESTARES, COMEDORES, } \\
\text { DORMITORIOS. } 200 \text { LUX: PASILOS. } 300 \text { LUX: } \\
\text { COCINA, LAVANDERO, SANITARIOS }\end{array}$ & NO CUMPLE & 0 & $\begin{array}{c}\text { CUMPLE CON } \\
\text { ILUMINACIÓNY } \\
\text { NORMAS MÍNIMAS } \\
\end{array}$ & 1 & $\begin{array}{c}\text { TOMASE } \\
\text { ILUMINACIÓN > AL } \\
\text { MÍNIMO } \\
\end{array}$ & 2 & & & 1 & 1 & 2 \\
\hline 25 & INSTALACIONES SANITARIAS & $\begin{array}{c}\text { PARAA } \\
\text { BLANCAS. YA } \\
\text { NEGRAS. }\end{array}$ & $\begin{array}{l}\text { TANQUE EN VIVIENDA; AGUA CALIENTE; } \\
\text { CLOACAS }\end{array}$ & NO TIENE & 0 & CLOACAS & 1 & $\begin{array}{l}\text { CLOACASYAGUA } \\
\text { CALIENTE }\end{array}$ & 2 & $\begin{array}{l}\text { CLOACAS, AGUA } \\
\text { CALIENTEY } \\
\text { TANQUE } \\
\end{array}$ & 3 & 1 & 1 & 3 \\
\hline 26 & DRENAJES DE AGUAS DE LLUVA & NO & $\begin{array}{l}\text { DRENAJES DE TECHO; DRENAJES RETIRO; } \\
\text { DRENAJE PATIOS. }\end{array}$ & \begin{tabular}{|c|} 
NO HAY \\
PREVISIÓN \\
DE \\
DRENAJES \\
PARA AGUAS \\
DE \\
WUVA \\
\end{tabular} & 0 & \begin{tabular}{|c|} 
LOS TECHOS \\
DRENAN HASTA LA \\
CALEE
\end{tabular} & 1 & TECHOSYRETIROS & 2 & $\begin{array}{l}\text { TECHOS, REIIROSY } \\
\text { FUTUROS PATIOS }\end{array}$ & 3 & 1 & 1 & 3 \\
\hline 27 & OTROS SERVICIOS & NO & TELÉFONO, RECOLECCIÓN BASURA & NO HAY & 0 & \begin{tabular}{|c|} 
RECOLECCIÓN DE \\
BASURA MÍNIMO \\
UNA VEZ POR \\
SEMANA \\
\end{tabular} & 1 & $\begin{array}{l}\text { RECOLECCIÓNDE } \\
\text { BASURA MÁS } \\
\text { TELÉFONO }\end{array}$ & 2 & \begin{tabular}{|l} 
RECOLECCIÓNDE \\
BASURA, TELÉFONO.
\end{tabular} & 3 & 1 & 1 & 3 \\
\hline 28 & $\begin{array}{l}\text { SISTEMA CONSTRUCTIVO } \\
\text { EFICIENCIA DE MATERIALES }\end{array}$ & NO & $\begin{array}{l}\text { MATERIALES DURADEROS, AISLAMIENTO } \\
\text { TÉRMICO, SONIDO, IMPACTO. }\end{array}$ & & & \begin{tabular}{|c|} 
TECHOS DE \\
LÁMINAS \\
METÁLICAS, \\
MACHMBRADO DE \\
MADERA Y TEJA \\
ASFALTICA \\
\end{tabular} & 1 & $\begin{array}{c}\text { TECHOS } \\
\text { RESISTENTES, } \\
\text { CONCRETO, } \\
\text { LOSACERO, ISOTEX, } \\
\text { TABELONES. }\end{array}$ & 2 & $\begin{array}{l}\text { TECHOS } \\
\text { RESISTENIES, } \\
\text { PAREDES CON } \\
\text { ACABADOS } \\
\text { DURAREROS, } \\
\text { LADRIUO. } \\
\end{array}$ & 3 & 1 & 1 & 3 \\
\hline 29 & PRIVACIDAD EXTERNA E INTERNA & NO & $\begin{array}{c}\text { REIIROS } \geq 3 \text { MTS. PUERTAS DE SANITARIOS } \\
\text { NO ABREN HACIA ESTARES O COMEDOR. } \\
\text { REGISTRO VISUAL }\end{array}$ & NO CUMPLE & 0 & $\begin{array}{l}\text { RETIROS DE } \\
\text { FRENTEY } \\
\text { LATERALES >3M }\end{array}$ & 1 & $\begin{array}{l}\text { RETIROS } \geq 3 M T S, \\
\text { PUERTAS } \\
\text { SANITARIAS NO } \\
\text { ABREN SOBRE } \\
\text { ESTARESY } \\
\text { COMEDORES. } \\
\end{array}$ & 2 & $\begin{array}{l}\text { RETIROS } \geq 3 \text { 3MS, } \\
\text { PUERTAS } \\
\text { SANITARIAS NO } \\
\text { ABREN SOBRE } \\
\text { ESTARESY } \\
\text { COMEDORES. SIN } \\
\end{array}$ & 3 & 1 & 1 & 3 \\
\hline
\end{tabular}




\begin{tabular}{|c|c|c|c|c|c|c|c|c|c|c|c|c|c|c|}
\hline & & & & & & & & & & REGISTRO VISUAL & & & & \\
\hline 30 & $\begin{array}{l}\text { APARIENCIA EXTERNA DE LA } \\
\text { VIVENDA }\end{array}$ & NO & $\begin{array}{l}\text { UNIDAD: TIPOLOGÍA DE ABERTURASY } \\
\text { MATERIALES. MATERIALES RESISTENTES. } \\
\text { CLARIDAD DE LECTURA }\end{array}$ & $\begin{array}{l}\text { NOTIENE } \\
\text { UNIDAD }\end{array}$ & 0 & $\begin{array}{l}\text { UNIDADDE } \\
\text { FACHADASY } \\
\text { TECHOS }\end{array}$ & 1 & $\begin{array}{l}\text { UNIDAD MÁS } \\
\text { MATERIALES } \\
\text { RESISTENTES }\end{array}$ & 2 & & & 1 & 1 & 2 \\
\hline 31 & $\begin{array}{l}\text { TRATAMIENTO DE AREAS } \\
\text { EXTERIORES }\end{array}$ & NO & $\begin{array}{l}\text { ACERAS PERIMETRALES, ACCESO } \\
\text { PAVIMENTADO A LA VIVIENDA, } \\
\text { ESTACIONAMIENTO PAVIMENTADO }\end{array}$ & NO TIENE & 0 & $\begin{array}{c}\text { ACCESO } \\
\text { PAVIMENTADO A } \\
\text { VIVIENDA }\end{array}$ & 1 & $\begin{array}{c}\text { ACCESO } \\
\text { PAVIMENTADO A } \\
\text { VIVIENDA MÁS } \\
\text { ACERAS } \geq 20 \text { CMS. }\end{array}$ & 2 & $\begin{array}{c}\text { ACCESOY } \\
\text { ESTACIONAMIENTO } \\
\text { PAVIMENTADO A } \\
\text { VIVENDA MÁS } \\
\text { ACERAS } \geq 20 \text { CMS } \\
\end{array}$ & 3 & 1 & 1 & 3 \\
\hline 32 & $\begin{array}{l}\text { SEGURIDADY RIESGO DE LA } \\
\text { CONSTRUCCIÓN }\end{array}$ & $\begin{array}{l}\text { ESTRUCT. Y } \\
\text { SISMO }\end{array}$ & $\begin{array}{l}\text { NORMAS ESTRUCTURALES DE SISMO. } \\
\text { MATERIALES NOCIVOS O COMBUSTIBLES; } \\
\text { INUNDACIONES (ELEVACION SOBRE LA } \\
\text { CALE); DESLIZAMIENTOS }\end{array}$ & \begin{tabular}{|c|} 
SIN \\
PREVISIONES \\
ANTISISMICA \\
S O \\
UTIUZACIÓN \\
DE \\
MATERIALES \\
NOCIVOS \\
\end{tabular} & 0 & $\begin{array}{l}\text { CUMPLE TODAS } \\
\text { LAS NORMASY } \\
\text { MATERIALES DE } \\
\text { SEGURIDAD. }\end{array}$ & 1 & \begin{tabular}{|c|} 
CUMPLE TODAS LAS \\
NORMASY \\
MATERIALES DE \\
SEGURIDAD MÁS \\
ELEVACIÓN $\geq 20$ CMS \\
SOBRE LA CALE
\end{tabular} & 2 & & & 1 & 1 & 2 \\
\hline 33 & $\begin{array}{l}\text { SEGURIDAD CONTRA ROBO, } \\
\text { VANDALISMO, ACCESO DE } \\
\text { ANIMALES }\end{array}$ & NO & $\begin{array}{c}\text { PROTECCIÓN VENTANAS, PUERTAS, CERCA } \\
\text { EXTERIOR }\end{array}$ & \begin{tabular}{c|} 
NINGUNA \\
PROTECCIÓN
\end{tabular} & 0 & $\begin{array}{l}\text { PROTECCIÓNEN } \\
\text { VENTANAS O } \\
\text { PUERTAS }\end{array}$ & 1 & $\begin{array}{l}\text { PROTECCIÓNEN } \\
\text { VENTANASY } \\
\text { PUERTAS }\end{array}$ & 2 & $\begin{array}{l}\text { PROTECCIÓNEN } \\
\text { VENTANASY } \\
\text { PUERTAS MAS } \\
\text { CERCA EXTERIOR } \\
\end{array}$ & 3 & 1 & 2 & 3 \\
\hline
\end{tabular}

\section{ENTORNO Y LOCALIZACIÓN}

\begin{tabular}{|c|c|c|c|c|c|c|c|c|c|c|c|c|c|}
\hline 34 & $\begin{array}{c}\text { DISTANCIA A SERVICIOS ESCOLARES, MATERNALES, } \\
\text { PREESCOLARES, ESCUELAS }\end{array}$ & NO & DISTACIA $<400 \mathrm{M}$ & NO EXISTEN & 0 & EXISTE A DISTANCIA & $>400 \mathrm{M}$ & 1 & $\begin{array}{l}\text { EXISTE A DISTANCIA }< \\
400 \mathrm{M}\end{array}$ & 2 & 2 & 2 & 4 \\
\hline 35 & DISTANCIA A PARQUES Y CANCHAS DEPORTIVAS & NO & DISTACIA $<400 \mathrm{M}$ & NO EXISTEN & 0 & EXISTE A DISTANCIA & $>400 \mathrm{M}$ & 1 & $\begin{array}{c}\text { EXISTE A DISTANCIA }< \\
400 \mathrm{M}\end{array}$ & 2 & 2 & 2 & 4 \\
\hline 36 & DISTANCIA A COMERCIOS LOCALES & NO & $\begin{array}{c}\text { DISTACIA < 400M: } \\
\text { ABASTOS, } \\
\text { FARMACIAS, } \\
\text { PANADERIAS }\end{array}$ & NO EXISTEN & 0 & EXISTE A DISTANCIA & $>400 \mathrm{M}$ & 1 & $\begin{array}{l}\text { EXISTE A DISTANCIA }< \\
400 \mathrm{M}\end{array}$ & 2 & 2 & 2 & 4 \\
\hline 37 & DISTANCIA A AMBULATORIO & NO & DISTACIA $<400 \mathrm{M}$ & NO EXISTEN & 0 & EXISTE A DISTANCIA & $>400 \mathrm{M}$ & 1 & $\begin{array}{l}\text { EXISTE A DISTANCIA }< \\
400 \mathrm{M}\end{array}$ & 2 & 2 & 2 & 4 \\
\hline 38 & DISTANCIA A PARADA DE TRANSPORTE PÚBபCO & NO & DISTACIA $<400 \mathrm{M}$ & NO EXISTEN & 0 & EXISTE A DISTANCIA & $>400 \mathrm{M}$ & 1 & $\begin{array}{l}\text { EXISTE A DISTANCIA }< \\
400 \mathrm{M}\end{array}$ & 2 & 2 & 2 & 4 \\
\hline & & & & SUBTOTALES & & & & & & & & 48 & 121 \\
\hline
\end{tabular}


Aplicando la metodología de evaluación se obtuvieron ponderaciones para cada uno de los factores de los indicadores considerando las características de las viviendas de interés social en Manabí, la cual es la que viene realizando desde hace varias décadas el Estado por medio del brazo ejecutor del MIDUVI con el aun inalcanzable objetivo de solventar el déficit habitacional.

En la tipología de la vivienda se obtuvo una ponderación de 1 de 3 , mientras que en superficie del lote la ponderación fue de 3 de 3 , igualmente en el ancho del lote, el área de construcción tubo una ponderación de 2 de 3 , en cuanto a los accesos peatonales se obtuvo una ponderación de 1 de 3, así mismo en lo correspondiente a los estacionamientos, en el indicador de crecimientos se pondero con una puntuación de 1 de 3 , en los componentes especiales se obtuvo el mismo puntaje, mientras que en estares separados se lo puntuó con 1 de 3 , en los comedores separados la ponderación fue también de 1 de 3, así mismo en estares y comedores separados, de igual manera en el área de servicio de lavadero.
Mientras que la calificación de dormitorio principal fue de 1 de 3 , de la misma manera en dormitorios dobles e individuales, en sanitarios su ponderación fue de 1, la misma calificación en áreas de almacenaje, siendo lo mismo para el depósito de basura, así como también en las alturas interiores, la iluminación natural también fue de 1 , así mismo en lo que corresponde al asolamiento de los estaciones la ventilación natural las protecciones ambientales, la iluminación y tomas, la ponderación de instalaciones sanitarias también fue de 1 , en cuanto al drenaje de aguas Iluvias, el acceso a otros servicios fue de $1 \mathrm{~m}$ sistemas constructivos eficiencia de materiales llega tan solo a un puntaje 1.

En lo meritorio a privacidad externa la ponderación fue de 1 , la apariencia externa alcanzo una calificación de 1 , el tratamiento de áreas exteriores es de 1 de 3 , la seguridad de riesgo de la construcción es de 1 sobre 3 , la seguridad contra robo logra una ponderación de 1 sobre 3.

Por otra parte, en lo referente a entorno y localización la distancia a servicios escolares alcanza una 
ponderación de 2 sobre 2 , así mismo la distancia a parques y canchas deportivas alcanza la misma puntuación de 2 sobre 2 , la distancia a comercios locales es igual de 2 sobre 2, la distancia a centros ambulatorios también fue de 2 sobre 2 y finalmente la distancia a paradas de servicio de transporte público es de 2 de 2 .

De lo cual se obtuvo una puntuación final de 48 de 121 puntos lo que corresponde a un $39,6 \%$ de habitabilidad de un $100 \%$ posible; lamentablemente esta calificación de habitabilidad de la vivienda de interés social es totalmente real, la gran mayoría de iniciativas emprendidas por el MIDUVI para la creación de vivienda social realmente se traducen en la dotación de viviendas mínimas lejos de una habitabilidad deseada o medianamente óptima para albergar de forma satisfactoria a una familia tipo.

Según el programa "casa para todos" el ultimo emprendido para esta finalidad se plantea una vivienda $49 \mathrm{~m} 2$ lo cual es insuficiente para garantizar una buena habitabilidad de la vivienda, situación que se comprueba por la calificación alcanzada mediante la aplicación de ficha de indicadores a este tipo de vivienda.

La habitabilidad se cumple cuando las circunstancias que se instituyen como correctos o anheladas para el grupo familiar y su adecuada interacción (Bernal, 2015).

\section{Conclusiones}

La habitabilidad se constituye en las características que otorgan a un espacio físico la capacidad para brindar seguridad y confort a sus ocupantes de tal manera que puedan llevar a cabo su día a día de manera amena, situación que no se puede aplicar actualmente a las viviendas de interés social que se implementan en la provincia de Manabí.

La vivienda de interés social es un medio para potencializar el desarrollo urbano planificado mediante casas dotadas de seguridad, confort y seguridad, salubridad, que a la vez posean cercanía a parques, instituciones educativas y centros de salud.

La vivienda social de Manabí requiera mejorar su calidad, para 
convertirse una solución eficiente, de acuerdo a las necesidades de la familia tipo que a menudo está conformada por un promedio de 5 integrantes, propiciando la incorporación de sus habitantes en el desarrollo, selección, e implementación de las viviendas siendo así una solución tanto de habitabilidad como en la creación de plazas de trabajo en el sector de la construcción.

Una vez realizada la aplicación de la evaluación de cada uno de los indicadores de habitabilidad de la vivienda de interés social en la provincia de Manabí se obtuvo una puntuación final de 48 de 121 puntos lo que corresponde a un $39,6 \%$ de habitabilidad de un 100\% posible, situación que invita a tomar cartas en el asunto por parte del MIDUVI y de organismos relacionados al sector de la construcción en el Ecuador.

\section{Bibliografía}

Asamblea General de las Naciones Unidas. (s.f.). Conferencia Mundial de Derechos Humanos (1993), Declaración y Programa de Acción de Viena: ".

Asamblea Nacional. (2008). Constitución Política del
Ecuador.

Montecristi:

Asamblea

Nacional

Constituyente.

Bernal. (2015). Habitabilidad y correspondencia con la vivienda de inter social. México: Aguilar.

BID. (2012). Déficit habitacional en América Latina y El Caribe. Ginebra: Banco Interamericano de Desarrollo.

Bohorque, N. (2017). Estudio de viviendas prefabricadas para el cantón guayaquil, aplicando el marketing social. Guayaquil: Universidad de Guayaquil.

Britto, F. (2019). Diseño arquitectónico, selección de materiales ecológicos y construcción de vivienda biosustentable para estratos 1,2 y 3. Barranquilla: Universidad de la Costa.

Dalongaro, C. (2014). La satisfacción del cliente con las edificaciones. Argentina: Universidad Nacional de La Plata.

Fermín. (2015). Características fundamentales de la vivienda de interés social. Cali: Publicaciones JLV.

Mercado, S. J. (2015). Evaluación psico-scoail de la vivienda de interés social. México: Urbanismo en la era moderna.

Molar, M., \& Aguirre, L. (2013). ¿Cómo es la habitabilidad en viviendas de interés social? 
Caso de estudio: fraccionamientos Lomas Del Bosque y Privadas la Torre En Saltillo, Coahuila. Coahuila: Revista lberoamericana de las Ciencias Sociales y Humanísticas ISSN: 23957972.

Naula, E. (2018). Funcionalidad de los espacios interiores de la vivienda social en Ecuador. Ambato: Universidad Técnica de Ambato - Facultad de diseño arquitectura y arte.

ONU. (2020). Conferencia de Naciones Unidas sobre asentamientos humanos. Estambul - Turquía: Organización de las Naciones Unidas.

Ortiz. (2017). Elementos fundamentales para el confort del grupo familia. Perú: El Sol.

Rendón, V. (2019). Medición de la habitabilidad en la vivienda de interés social. Caso de estudio: Municipio de
Ecatepec y Tecámac. México: Publicación Semestral Pädi Vol. 7 No. 14 (2020) 59-65.

Rodas, A. (2018). La habitabilidad en la vivienda social en Ecuador a partir de la visión de la complejidad: elaboración de un sistema de análisis. Ecuador: Universidad Autónoma del Estado de Morelos.

Ultimahora. (2016). Aplicación de construcción tipo lego y su importancia. Ultimahora, págs. 45-46.

Véliz, J. (2019). Evolución de la vivienda de interes social en Portoviejo. Portoviejo: Universidad Técnica de Manabí. 